\title{
Probing Quark-Gluon Interactions with Transverse Polarized Scattering
}

K. Slifer, ${ }^{1,2}$ O. A. Rondón, ${ }^{1}$ A. Aghalaryan, ${ }^{3}$ A. Ahmidouch, ${ }^{4}$ R. Asaturyan, ${ }^{3}$ F. Bloch, ${ }^{5}$ W. Boeglin,,${ }^{6}$ P. Bosted, ${ }^{7}$ C. Carasco, ${ }^{5}$ R. Carlini, ${ }^{7}$ J. Cha, ${ }^{8}$ J. P. Chen, ${ }^{7}$ M. E. Christy, ${ }^{9}$ L. Cole, ${ }^{9}$ L. Coman,,${ }^{6}$ D. Crabb, ${ }^{1}$ S. Danagoulian, ${ }^{4}$ D. Day, ${ }^{1}$ J. Dunne,${ }^{8}$ M. Elaasar, ${ }^{10}$ R. Ent, ${ }^{7}$ H. Fenker, ${ }^{7}$ E. Frlez, ${ }^{1}$ D. Gaskell, ${ }^{7}$ L. Gan, ${ }^{11}$ J. Gomez, ${ }^{7}$ B. Hu, ${ }^{9}$ J. Jourdan, ${ }^{5}$ M. K. Jones, ${ }^{7}$ C. Keith, ${ }^{7}$ C. E. Keppel, ${ }^{9}$ M. Khandaker, ${ }^{12}$ A. Klein, ${ }^{13}$ L. Kramer, ${ }^{6}$ Y. Liang, ${ }^{9}$ J. Lichtenstadt, ${ }^{14}$ R. Lindgren, ${ }^{1}$ D. Mack, ${ }^{7}$ P. McKee, ${ }^{1}$ D. McNulty, ${ }^{1,15}$ D. Meekins, ${ }^{7}$ H. Mkrtchyan, ${ }^{3}$ R. Nasseripour, ${ }^{6}$ I. Niculescu, ${ }^{7}$ K. Normand, ${ }^{5}$ B. Norum, ${ }^{1}$ D. Pocanic, ${ }^{1}$ Y. Prok, ${ }^{1}$ B. Raue, ${ }^{6}$ J. Reinhold,${ }^{6}$ J. Roche, ${ }^{7}$ D. Kiselev, ${ }^{5,16}$ N. Savvinov, ${ }^{17}$ B. Sawatzky, ${ }^{1}$ M. Seely, ${ }^{7}$ I. Sick, ${ }^{5}$ C. Smith, ${ }^{1}$ G. Smith, ${ }^{7}$ S. Stepanyan, ${ }^{18}$ L. Tang, ${ }^{9}$ S. Tajima, ${ }^{1}$ G. Testa, ${ }^{5}$ W. Vulcan, ${ }^{7}$ K. Wang, ${ }^{1}$ G. Warren,${ }^{5,7}$ F. R. Wesselmann, ${ }^{1,12}$ S. Wood, ${ }^{7}$ C. Yan, ${ }^{7}$ L. Yuan, ${ }^{9}$ J. Yun, ${ }^{19}$ M. Zeier, ${ }^{1}$ and H. Zhu ${ }^{1}$

(Resonance Spin Structure Collaboration)

\author{
${ }^{1}$ University of Virginia, Charlottesville, Virginia 22903, USA \\ ${ }^{2}$ University of New Hampshire, Durham, New Hampshire 03824, USA \\ ${ }^{3}$ Yerevan Physics Institute, Yerevan, Armenia 0036 \\ ${ }^{4}$ North Carolina A\&T State University, Greensboro, North Carolina 27411, USA \\ ${ }^{5}$ Universität Basel, CH-4056 Basel, Switzerland \\ ${ }^{6}$ Florida International University, Miami, Florida 33199, USA \\ ${ }^{7}$ Thomas Jefferson National Accelerator Facility, Newport News, Virginia 23606, USA \\ ${ }^{8}$ Mississippi State University, Mississippi State, Mississippi 39762, USA \\ ${ }^{9}$ Hampton University, Hampton, Virginia 23668, USA \\ ${ }^{10}$ Southern University at New Orleans, New Orleans, Louisiana 70126, USA \\ ${ }^{11}$ University of North Carolina, Wilmington, North Carolina 28403, USA \\ ${ }^{12}$ Norfolk State University, Norfolk, Virginia 23504, USA \\ ${ }^{13}$ Old Dominion University, Norfolk, Virginia 23529, USA \\ ${ }^{14}$ Tel Aviv University, Tel Aviv, 69978 Israel \\ ${ }^{15}$ University of Massachusetts, Amherst, Massachusetts 01003, USA \\ ${ }^{16}$ Paul Scherrer Institut, Villigen, Switzerland \\ ${ }^{17}$ University of Maryland, College Park, Maryland 20742, USA \\ ${ }^{18}$ Kyungpook National University, Daegu 702-701, South Korea \\ ${ }^{19}$ Virginia Polytechnic Institute \& State University, Blacksburg, Virginia 24061, USA
}

(Received 15 August 2009; revised manuscript received 24 August 2010; published 2 September 2010)

We have extracted QCD matrix elements from our data on doubly polarized inelastic scattering of electrons on nuclei. We find the higher twist matrix element $\tilde{d}_{2}$, which arises strictly from quark-gluon interactions, to be unambiguously nonzero. The data also reveal an isospin dependence of higher twist effects if we assume that the Burkhardt-Cottingham sum rule is valid. The fundamental Bjorken sum rule obtained from the $a_{0}$ matrix element is satisfied at our low momentum transfer.

Polarized deep inelastic scattering (DIS) of leptons on nucleons has helped to establish the modern understanding of nucleon structure. Measurements of the longitudinal nucleon spin structure function (SSF) $g_{1}$ in inclusive doubly polarized DIS, where only the final lepton is detected, have revealed that the nucleon spin does not result from the naive sum of the spins of its three valence quarks. Rather, contributions from sea quarks, gluon spin and orbital angular momentum seem to be required [1-3]. DIS is well understood at high energies, when the virtual photons exchanged between the incident beam and the target illuminate the nucleon constituents as the asymptotically free quarks of QCD. This is the regime of small separations between quarks and gluons, which is best tested with short wavelength electromagnetic probes. The processes that polarized DIS explores are dominated by leading order terms in the expansion of $g_{1}$ that are related to the noninteracting partonic description of QCD. However, for a full understanding, the interactions among nucleon constituents must also be probed by looking into contributions beyond leading order, which grow in importance at lower energies. Of particular interest are the so called "higher twist" terms, which measure correlations among partons (quarks and gluons), and offer unique perspectives on the phenomenon of confinement inside nucleons and other hadrons.

Access to these subleading interactions is possible with transverse polarized inelastic scattering. In this configuration, the target spin is held orthogonal to the incident lepton 
spin, and the $g_{2}$ spin structure function dominates compared to $g_{1}$ [4]. QCD matrix elements that arise from multiparton correlations can be extracted directly from moments of $g_{2}$. However, this approach has been limited due to the technical challenges involved in aligning proton spins at $90^{\circ}$ relative to the beam. As we detail below, we have carried out a complete measurement of the longitudinal and transverse inclusive SSFs $g_{1}$ and $g_{2}$ of the proton and deuteron, with the goal of extracting matrix elements related to quark-gluon, $q g$, interactions.

The operator product expansion [5] relates the moments of the SSFs to reduced quark and gluon matrix elements representing the multiparton correlations that lead to confinement. This perturbative approach to QCD is formulated in terms of the Cornwall-Norton (CN) moments [6]:

$$
\Gamma_{1,2}^{(n)}\left(Q^{2}\right)=\int_{0}^{1} d x x^{n-1} g_{1,2}\left(x, Q^{2}\right)
$$

where $x=Q^{2} / 2 M \nu$ is the Bjorken scaling variable, $Q^{2}$ is the four-momentum transfer squared of the scattering process, $M$ the nucleon mass, and $\nu$ the energy transfer. Conventionally, the index $n=1$ is not displayed.

In the operator product expansion, the $\mathrm{CN}$ moments are expanded in a power series in $1 / Q^{(\tau-2)}$ of increasing "twist" $\tau$, which is defined as the mass dimension minus the spin $n$ of the corresponding QCD operator [5]. The leading twist-2 terms map to the successful predictions of the parton model, such as the $Q^{2}$ independence of the structure functions up to logarithmic corrections. Higher twist (HT) corrections arise from the nonperturbative multiparton interactions, whose contributions at low energy increase as $1 / Q^{\tau}$, reflecting confinement. Specifically, twist-3 represents $q g q$ correlations, so a nonzero result at a given $Q^{2}$ for the term representing twist-3 signals a departure from the noninteracting partonic regime.

Studies of higher twist effects [7-11] have typically focused on the $\mathrm{CN}$ moments $I\left(Q^{2}\right)=\int_{0}^{1} d x x^{2}\left(2 g_{1}+\right.$ $\left.3 g_{2}\right)$ and $\Gamma_{1}\left(Q^{2}\right)$, in order to extract the twist-3 and twist4 matrix elements. However, as recently stressed in $[6,12]$, this approach is only appropriate when terms of purely kinematic origin, known as "target mass corrections" (TMCs) [13-18] for their connection to the finite nucleon mass, can be neglected. These terms, of order $\mathcal{O}\left(M^{2} / Q^{2}\right)$, are formally related to twist-2 operators and must be cleanly separated from the desired dynamical higher twists. $I\left(Q^{2}\right)$ is often labeled " $d_{2}$ " in the literature, although, as discussed below, it is not equivalent to the HT matrix element indicated by the same symbol.

As emphasized in [12], dynamical HT can be extracted to order $\mathcal{O}\left(M^{8} / Q^{8}\right)$ by using Nachtmann moments [16,19], which depend on the scaling variable $\xi=2 x /(1+$ $\left.\sqrt{1+(2 x M)^{2} / Q^{2}}\right)$. This variable extends scaling as $x$ approaches 1 , for data measured at low $Q^{2}$. The Nachtmann moments are defined as

$$
\begin{aligned}
M_{1}^{n}\left(Q^{2}\right) \equiv & \frac{1}{2} \tilde{a}_{n-1} \equiv \frac{1}{2} a_{n} E_{1}^{n} \\
= & \int_{0}^{1} \frac{d x}{x^{2}} \xi^{n+1}\left[\left\{\frac{x}{\xi}-\frac{n^{2}}{(n+2)^{2}} \frac{M^{2} x \xi}{Q^{2}}\right\} g_{1}\right. \\
& \left.-\frac{4 n}{n+2} \frac{M^{2} x^{2}}{Q^{2}} g_{2}\right], \\
M_{2}^{n}\left(Q^{2}\right) \equiv & \frac{1}{2} \tilde{d}_{n-1} \equiv \frac{1}{2} d_{n} E_{2}^{n} \\
= & \int_{0}^{1} \frac{d x}{x^{2}} \xi^{n+1}\left[\frac{x}{\xi} g_{1}+\left\{\frac{n}{n-1} \frac{x^{2}}{\xi^{2}}-\frac{n}{n+1} \frac{M^{2} x^{2}}{Q^{2}}\right\} g_{2}\right] .
\end{aligned}
$$

Here, the $a_{n}\left(d_{n}\right)$ represent twist-2 (-3) matrix elements, while the $E_{2}^{n}$ are the corresponding Wilson coefficients, which contain logarithmic QCD corrections. For convenience, these corrections are absorbed into the definition of the effective matrix elements $\tilde{a}_{n}$ and $\tilde{d}_{n}$. The index runs over $n=1,3, \ldots$ for $M_{1}$, and $n=3,5, \ldots$ for $M_{2}$. When $Q^{2} \gg M^{2}$, the Nachtmann moments simplify:

$$
M_{1}^{1}\left(Q^{2}\right) \rightarrow \Gamma_{1}\left(Q^{2}\right), \quad 2 M_{2}^{3}\left(Q^{2}\right) \rightarrow I\left(Q^{2}\right) .
$$

Consequently, deviations from unity of the ratio of Nachtmann to $\mathrm{CN}$ moments give a quantitative measure of the TMCs at finite $Q^{2}$ :

$$
R_{1}\left(Q^{2}\right)=\frac{M_{1}^{1}\left(Q^{2}\right)}{\Gamma_{1}\left(Q^{2}\right)}, \quad R_{2}\left(Q^{2}\right)=\frac{2 M_{2}^{3}\left(Q^{2}\right)}{I\left(Q^{2}\right)} .
$$

The leading twist- 2 contribution to $g_{2}$ can be determined from $g_{1}$ via the Wandzura-Wilczek relation [20]:

$$
g_{2}^{\mathrm{ww}}\left(x, Q^{2}\right) \equiv-g_{1}\left(x, Q^{2}\right)+\int_{x}^{1} y^{-1} g_{1}\left(y, Q^{2}\right) d y .
$$

So any twist- 3 effects arise entirely from $\bar{g}_{2}=g_{2}-g_{2}^{\mathrm{ww}}$.

Experiment E01-006 was conducted in Hall $\mathrm{C}$ of the Thomas Jefferson National Accelerator Facility by the Resonance Spin Structure (RSS) collaboration. We measured the parallel and perpendicular double spin asymmetries $A_{\|}$and $A_{\perp}$ in the scattering of $100 \mathrm{nA}$ polarized $5.755 \mathrm{GeV}$ electrons on polarized protons and deuterons. Scattered electrons were detected at an angle of $13.15^{\circ}$ using the Hall C High Momentum Spectrometer. The kinematic coverage in invariant mass was $1.090<W<$ $1.910 \mathrm{GeV}$, corresponding to $x_{0}=0.316<x<x_{\max }=$ 0.823 , at an average four-momentum transfer of $\left\langle Q^{2}\right\rangle=$ $1.28 \pm 0.21 \mathrm{GeV}^{2}$. Systematic uncertainties are detailed in Table I, with more details in $[21,22]$.

The SSFs were extracted from the measured asymmetries using a fit to the ratio of longitudinal to transverse cross sections $R$ [23] and the unpolarized structure function $F_{1}$ [23], based primarily on data measured previously in Hall C. The standard formulas and procedure used to obtain $A_{\|}, A_{\perp}$ and the SSFs from the data are detailed in [1,21]. Simultaneous determination of both SSFs 
TABLE I. Measured integral systematic uncertainties (in \%) arising from the target dilution factor $f$, radiative corrections (RC), the $F_{1}$ and $R$ data fits, beam and target polarizations $P_{b}$ and $P_{t}$, and the evolution of our data fit to constant $Q^{2}$.

\begin{tabular}{lllll}
\hline \hline & $\Gamma_{1}^{p}$ & $\Gamma_{1}^{d}$ & $\tilde{d}_{2}^{p}$ & $\tilde{d}_{2}^{d}$ \\
\hline$f$ & 4.9 & 5.1 & 4.9 & 5.1 \\
$\mathrm{RC}$ & 3.2 & 4.0 & 9.5 & 4.5 \\
$F_{1}$ & 3.0 & 4.0 & 3.0 & 4.0 \\
$R$ & 0.9 & 1.8 & 1.2 & 2.8 \\
$P_{b} P_{t}$ & 1.6 & 1.6 & 5.2 & 3.1 \\
$Q^{2}$ dependence & 0.33 & 0.1 & 4.3 & 0.3 \\
Total & 6.8 & 8.0 & 13.0 & 8.9 \\
\hline \hline
\end{tabular}

allowed us to evaluate the moments without model input for $g_{2}$, as was necessary in some previous analyses [9].

The moments reported herein are evaluated at $\left\langle Q^{2}\right\rangle=$ $1.28 \mathrm{GeV}^{2}$, and have been decomposed into contributions from the measured resonance region $x_{0}<x<x_{\text {thr }}$ (labeled with " $m$ "), the well-known $x=1$ elastic ("el") contribution, and the unmeasured (" $u$ ") portion below $x_{0}$. We note that the small difference between our experimental $x_{\max }$ and the nucleon inelastic threshold $x_{\text {thr }}$ has negligible impact on the integrated results. We have used fits [21] to our data to evaluate the moments in the measured region. The nucleon elastic contribution was calculated using the form factor parameterizations of [24]. Relative uncertainties of $5 \%, 1 \%, 14 \%$, and $2.5 \%$ have been assumed for the electromagnetic form factors $G_{E}^{P}, G_{M}^{P}, G_{E}^{N}$, and $G_{M}^{N}$, respectively. For the deuteron, $x=1$ represents quasielastic scattering, the strength of which we estimate by combining the nucleon elastic contributions using the $D$-state correction discussed below. The deuteron nuclear elastic contribution is negligible here. We have also evaluated the neutron and nonsinglet (proton-neutron) moments by using the relation [25] $\Gamma^{n}=\Gamma^{\Sigma}-\Gamma^{p}$, where the singlet $\Gamma^{\Sigma}=$ $\Gamma^{d} / \gamma_{D}$, and $\gamma_{D} \simeq 0.926 \pm 0.016[26]$ is the $D$-state correction to the deuteron wave function. The uncertainty arising from this approach is estimated [27] to be $\mathcal{O}(1 \%)$. The singlet and nonsinglet results assume negligible heavy quark contributions.

Table II provides numerical values for the moments. The ratio $R_{2}$ differs significantly from unity, indicating large unwanted twist-2 kinematical contributions to $I\left(Q^{2}\right)$. The full results for the matrix element $\tilde{d}_{2}$ indicate clear twist- 3 at more than $6 \sigma$ for the proton and $3 \sigma$ for the neutron. These nonvanishing results unambiguously indicate the presence of $q g q$ correlations. Their magnitudes can be used in comparing with nucleon models.

The values of $I\left(Q^{2}\right)$ and $\tilde{d}_{2}$ for $x<x_{0}$ were estimated to be zero, with a systematic error described below. In this unmeasured region, $\xi \approx x$ and the $\mathrm{CN}$ and Nachtmann truncated moments converge, so there is little difference between $I\left(Q^{2}\right)$ and $\tilde{d}_{2} . I\left(Q^{2}\right)$ is expected to be small in this unmeasured region, because of the decreasing importance of higher twists at low $x$, and the strong suppression due to the $x^{2}$ weighting of the integral. This assumed low $x$ behavior is supported by our data. Figure 1 (left) displays the $x$ - dependence of the measured $g_{2}$ structure function. It is clear that $\bar{g}_{2}$ is nearly constant and consistent with zero within errors for the proton, near the low end of our measured range $x_{0}<x \leqq 0.37$. The deuteron data show a similar behavior, different from zero only at the one $\sigma$ (statistical) level. Neutron data [28] at $x \sim 0.2$ and similar $Q^{2}$ are also consistent with $\bar{g}_{2} \cong 0$. We take the error on

TABLE II. RSS Moments evaluated at $\left\langle Q^{2}\right\rangle=1.28 \mathrm{GeV}^{2}$. The ratio $R_{1}=M_{1}^{1} / \Gamma_{1}$ and $R_{2}=\tilde{d}_{2} / I$.

\begin{tabular}{|c|c|c|c|c|c|c|c|c|c|c|c|}
\hline \multirow{2}{*}{$x$-range } & & \multicolumn{3}{|c|}{ Proton } & \multicolumn{3}{|c|}{ Deuteron } & \multicolumn{2}{|c|}{ Neutron } & \multicolumn{2}{|c|}{ Non-Singlet } \\
\hline & & Value & Stat & Syst & Value & Stat & Syst & Value & Total & Value & Total \\
\hline \multirow{4}{*}{$\begin{array}{l}0<x<x_{0} \\
\text { (unmeasured) }\end{array}$} & $M_{1}^{1}$ & 0.0676 & $\cdots$ & 0.0069 & 0.0274 & $\cdots$ & 0.0104 & -0.0381 & 0.0132 & 0.1057 & 0.0178 \\
\hline & $\Gamma_{1}$ & 0.0683 & $\cdots$ & 0.0069 & 0.0280 & $\cdots$ & 0.0099 & -0.0381 & 0.0127 & 0.1065 & 0.0176 \\
\hline & $\tilde{d}_{2}$ & 0.0 & $\cdots$ & 0.0008 & 0.0 & $\cdots$ & 0.0013 & 0.0 & 0.0016 & 0.0 & 0.0021 \\
\hline & $I$ & 0.0 & $\cdots$ & 0.0008 & 0.0 & $\ldots$ & 0.0013 & 0.0 & 0.0016 & 0.0 & 0.0021 \\
\hline \multirow{4}{*}{$\begin{array}{l}x_{0} \leq x<x_{\mathrm{thr}} \\
\text { (measured) }\end{array}$} & $M_{1}^{1}$ & 0.0330 & 0.0005 & 0.0022 & 0.0290 & 0.0010 & 0.0023 & -0.0016 & 0.0036 & 0.0346 & 0.0054 \\
\hline & $\Gamma_{1}$ & 0.0351 & 0.0005 & 0.0024 & 0.0315 & 0.0011 & 0.0025 & -0.0010 & 0.0039 & 0.0361 & 0.0058 \\
\hline & $\tilde{d}_{2}$ & 0.0037 & 0.0004 & 0.0005 & 0.0048 & 0.0008 & 0.0004 & 0.0015 & 0.0012 & 0.0022 & 0.0016 \\
\hline & $I$ & 0.0057 & 0.0006 & 0.0007 & 0.0082 & 0.0013 & 0.0007 & 0.0031 & 0.0019 & 0.0026 & 0.0026 \\
\hline \multirow{4}{*}{$\begin{array}{l}x=1 \\
\text { (elastic) }\end{array}$} & $M_{1}^{1}$ & 0.0287 & $\cdots$ & 0.0006 & 0.0338 & $\cdots$ & 0.0012 & 0.0078 & 0.0005 & 0.0208 & 0.0008 \\
\hline & $\Gamma_{1}$ & 0.0351 & $\ldots$ & 0.0010 & 0.0373 & $\ldots$ & 0.0015 & 0.0051 & 0.0007 & 0.0300 & 0.0012 \\
\hline & $\tilde{d}_{2}$ & 0.0067 & $\ldots$ & 0.0008 & -0.0021 & $\cdots$ & 0.0010 & -0.0090 & 0.0007 & 0.0157 & 0.0011 \\
\hline & $I$ & 0.0306 & $\cdots$ & 0.0025 & 0.0088 & $\ldots$ & 0.0029 & -0.0212 & 0.0019 & 0.0518 & 0.0031 \\
\hline \multirow{4}{*}{$\begin{array}{l}0 \leq x \leq 1 \\
\text { (Total) }\end{array}$} & $M_{1}^{1}$ & 0.1293 & 0.0005 & 0.0073 & 0.0902 & 0.0010 & 0.0108 & -0.0318 & 0.0137 & 0.1611 & 0.0187 \\
\hline & $\Gamma_{1}$ & 0.1385 & 0.0005 & 0.0074 & 0.0968 & 0.0011 & 0.0103 & -0.0340 & 0.0134 & 0.1725 & 0.0185 \\
\hline & $\tilde{d}_{2}$ & 0.0104 & 0.0004 & 0.0013 & 0.0027 & 0.0008 & 0.0017 & -0.0075 & 0.0021 & 0.0179 & 0.0029 \\
\hline & $I$ & 0.0364 & 0.0006 & 0.0027 & 0.0170 & 0.0013 & 0.0032 & -0.0180 & 0.0031 & 0.0544 & 0.0045 \\
\hline \multirow[t]{2}{*}{$0 \leq x \leq 1$} & $R_{1}$ & 0.933 & 0.003 & & 0.932 & 0.012 & & 0.936 & 0.037 & 0.934 & 0.008 \\
\hline & $R_{2}$ & 0.286 & 0.014 & & 0.159 & 0.078 & & 0.415 & 0.045 & 0.329 & 0.025 \\
\hline
\end{tabular}




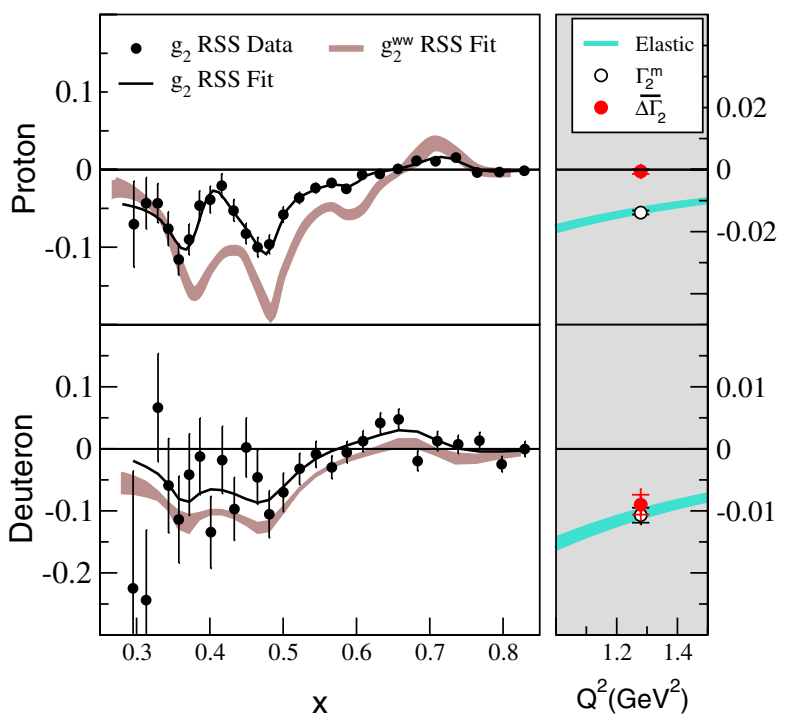

FIG. 1 (color online). Left top: RSS proton $g_{2}$ data [21], along with the RSS fit [21]. The shaded curve is $g_{2}^{\mathrm{ww}}$ evaluated from the RSS fit. Left bottom: RSS deuteron $g_{2}$ data, and similar curves as above. Right top: The open circle is the measured RSS proton $\Gamma_{2}^{m}\left(Q^{2}\right)$ data. The full circle is $\Delta \bar{\Gamma}_{2}\left(Q^{2}\right)$. The inner (outer) error bars represent statistical (total) uncertainty. The shaded curve is the elastic contribution to $\Gamma_{2}\left(Q^{2}\right)$. Right bottom: Same as above, but for deuteron.

$\bar{g}_{2}$, evaluated at $x_{0}, \delta \bar{g}_{2}$, as a conservative upper limit for the integrand of $I\left(Q^{2}\right)$ in the unmeasured low $x$ region. For the proton, we determined this upper limit by assuming a constant extrapolation of the value of $\delta \bar{g}_{2}\left(x_{0}\right)$ down to $x=0$. For the deuteron, we evaluated both a linear and constant extrapolation, averaged both assumptions and added quadratically one-half their difference as "model" error to the $\delta \bar{g}_{2}^{d}$ fit error to obtain the value in Table II. A divergence of $g_{2}$ as $x \rightarrow 0$ could invalidate this assumption. Such a possible divergence for $x \lessgtr 0.001$ was discussed in [29]. Normalizing the low $x$ dependence of $\bar{g}_{2}$ given in [29] to our $\delta \bar{g}_{2}$ estimates, we find the additional contribution to be negligible.

It is instructive to compare our twist-3 results to previous measurements. Stanford linear accelerator center (SLAC) experiment E155 [1] reported an evaluation of $I\left(Q^{2}\right)$ at $\left\langle Q^{2}\right\rangle=5 \mathrm{GeV}^{2}$. We have corrected those results for TMCs [12], to obtain $0.0028 \pm 0.0015$ and $0.0072 \pm 0.0044$ for the proton and neutron, respectively. For direct comparison, we performed a pQCD evolution [30] from RSS to the SLAC kinematics. At LO, we find $\tilde{d}_{2}^{p}=0.0021 \pm 0.0006$ and $\tilde{d}_{2}^{n}=0.0031 \pm 0.0038$, which are consistent with the E155 results. The elastic contribution [24] at these kinematics is smaller than the uncertainties and is not included in the results of this paragraph. Next-to-leadingorder (NLO) corrections [31] to our data have been calculated and will be discussed in a future publication.

Twist-3 effects also manifest in the first moment of $g_{2}$. The Burkhardt-Cottingham (BC) sum rule [32] predicts that $\Gamma_{2}$ vanishes for all $Q^{2}$. This sum rule can be derived from the unsubtracted dispersion relation for the virtual-virtual Compton scattering amplitudes, in an analogous fashion to the more famous Gerasimov-Drell-Hern (GDH) and Bjorken sum rules. It provides a remarkably clean tool to investigate nucleon structure, since it is free from both QCD radiative corrections and TMCs [33]. Initial measurements by the E155 collaboration found a $2 \sigma$ inconsistency with the proton $\mathrm{BC}$ sum rule at large $Q^{2}$, while the same group found the deuteron sum rule to hold. Later measurements $[3,7]$ at lower momentum transfer found agreement with both the neutron and $\mathrm{H}^{3} \mathrm{e} \mathrm{BC}$ sum rules.

The leading twist $g_{2}^{\mathrm{ww}}$ satisfies the BC sum rule exactly, so any violation must arise from higher twist effects. For convenience we split the full integral as:

$$
\Gamma_{2}=\Gamma_{2}^{\mathrm{ww}}+\bar{\Gamma}_{2}+\Gamma_{2}^{\mathrm{el}}=\bar{\Gamma}_{2}^{u}+\bar{\Gamma}_{2}^{m}+\Gamma_{2}^{\mathrm{el}},
$$

where the overbar signifies removal of the leading twist contribution, and we have made use of the fact that $\Gamma_{2}^{\mathrm{ww}} \equiv 0$ by definition. The elastic contribution is well known, as displayed in Fig. 1, and we can evaluate $\bar{\Gamma}_{2}^{m}$ directly from our own data. $\bar{\Gamma}_{2}^{u}$ is pure higher twist, and cannot be directly determined, due to the lack of low $x g_{2}$ data. However, the difference,

$$
\Delta \bar{\Gamma}_{2} \equiv \Gamma_{2}-\bar{\Gamma}_{2}^{u}
$$

depends only on measured data. A significant nonzero result for this quantity would indicate a higher twist contribution to the integral in the region $x<x_{0}$, assuming of course, that the BC sum rule holds. Table III indicates that the proton results are consistent with vanishing HT effects at low $x$. On the other hand, the singlet results indicate the need for significant HT contributions to $\Gamma_{2}$ at low $x$, if the $\mathrm{BC}$ sum rule is to be satisfied. This could indicate an isospin dependence of the $\mathrm{BC}$ sum rule, or alternatively, a modification to the sum rule due to nuclear effects.

Nachtmann moments are also useful for obtaining leading twist matrix elements free of TMCs. The $a_{0}$ nonsinglet matrix element corresponds to the lowest moment $\Gamma_{1}^{p-n}$ of

\begin{tabular}{|c|c|c|c|c|c|c|c|c|c|c|}
\hline & \multicolumn{3}{|c|}{ Proton } & \multicolumn{3}{|c|}{ Deuteron } & \multicolumn{2}{|c|}{ Neutron } & \multicolumn{2}{|c|}{ Non-Singlet } \\
\hline & Value & Stat & Syst & Value & Stat & Syst & Value & Total & Value & Total \\
\hline$\Gamma_{2}^{\mathrm{el}}$ & -0.0132 & $\cdots$ & 0.0004 & -0.0219 & $\cdots$ & 0.0009 & -0.0105 & 0.0005 & -0.0027 & 0.0007 \\
\hline$\Gamma_{2}^{m}$ & -0.0138 & 0.0007 & 0.0009 & -0.0107 & 0.0012 & 0.0009 & 0.0022 & 0.0020 & -0.0160 & 0.0028 \\
\hline $\bar{\Gamma}_{2}^{m}$ & 0.0126 & 0.0008 & 0.0020 & 0.0129 & 0.0016 & 0.0018 & 0.0013 & 0.0034 & 0.0113 & 0.0051 \\
\hline$\Delta \bar{\Gamma}_{2}$ & -0.0006 & 0.0008 & 0.0021 & -0.0090 & 0.0016 & 0.0020 & -0.0092 & 0.0035 & 0.0086 & 0.0051 \\
\hline
\end{tabular}

TABLE III. The higher twist $\Delta \bar{\Gamma}_{2}=\Gamma_{2}-\bar{\Gamma}_{2}^{u}$ evaluated at $\left\langle Q^{2}\right\rangle=1.28 \mathrm{GeV}^{2}$. 
the isovector nucleon $g_{1}$, which is related to the nucleon axial charge $g_{A}$ via the Bjorken sum rule [34]. This relation is a direct consequence of $\mathrm{QCD}$, and experimental tests $[1,2]$ of the sum rule have played a critical role in confirming QCD as the correct theory of the strong interaction. The target mass corrections to $\Gamma_{1}$ given by the ratio $R_{1}$ are small at our kinematics, due to a combination of dominant contributions from the region below our lowest measured $x_{0}$, and the nearly identical values of $x$ and $\xi$ in that region. It is interesting to note that $R_{1} \approx 93 \%$ for all targets, when the elastic contribution is included. The $M_{1}^{1}$ nonsinglet result including the elastic part $(0.161 \pm 0.019)$ is in excellent agreement with theory: Ref. [35] predicts 0.212 , which reduces to 0.155 after application of the next to nextto-leading order corrections [36] appropriate at our $Q^{2}$. For the $g_{1}$ integrals, the contribution from the $x<x_{0}$ region was calculated from Regge-inspired fits to SLAC $g_{1}^{p}$ and $g_{1}^{d}$ data within a band $Q^{2}=1.3 \pm 0.3 \mathrm{GeV}^{2}$. We find $g_{1}=a x^{b}(1-x)^{3}\left(1+c / Q^{2}\right)$ with $a=0.392 \pm 0.254$, $b=0.0676 \pm 0.084, c=0.0636 \pm 0.681$ for the proton, and $a=1.778 \pm 1.971, \quad b=0.739 \pm 0.407$, and $c=$ $-0.786 \pm 0.0256$ for the deuteron.

In summary, we have measured the spin structure function moments of the proton, deuteron, and neutron in the resonance region. The kinematic weighting of higher moments suppresses contributions from the low $x$ region, thus minimizing the systematic uncertainty of this unmeasured piece of the integral. Our analysis indicates a large TMC contamination of the $\mathrm{CN}$ moment $I\left(Q^{2}\right)$, which has often been used to determine the magnitude of higher twist effects. The alternate Nachtmann moment $\tilde{d}_{2}$ minimizes TMCs and allows a clean extraction of the twist-3 reduced matrix element. The previously undetermined $\tilde{d}_{2}$ provides unambiguous evidence of dynamical twist-3 effects, which arise from quark-gluon interactions. The first moment of $g_{2}$ indicates an isospin dependence of HT effects in the region of small Bjorken scaling variable, assuming that the $\mathrm{BC}$ sum rule holds. After nearly 30 years of spin-dependent investigations, the $g_{2}^{p}$ structure function remains largely unknown. But the data reported here and future analyses [37] will shed light on this fundamental nucleon observable.

We would like to thank the Hall $\mathrm{C}$ technical staff and the accelerator operators for their efforts and dedication. This work was supported by the Department of Energy, the National Science Foundation, the Schweizerische Nationalfonds, and by the Institute of Nuclear and Particle Physics of the University of Virginia. The Southeastern Universities Research Association operates the Thomas Jefferson National Accelerator Facility for the DOE under Contract No. DE-AC05-84ER40150, mod. \# 175.

[1] K. Abe (E143), Phys. Rev. D 58, 112003 (1998); P. L. Anthony (E155), Phys. Lett. B 493, 19 (2000); 553, 18 (2003).

[2] A. Airapetian et al., Phys. Rev. D 75, 012007 (2007).
[3] M. Amarian et al., Phys. Rev. Lett. 92, 022301 (2004).

[4] R. L. Jaffe, Comments Nucl. Part. Phys. 19, 239 (1990).

[5] B. Ehrnsperger, A. Schafer, and L. Mankiewicz, Phys. Lett. B 323, 439 (1994); J. Kodaira, Y. Yasui, and T. Uematsu, ibid. 344, 348 (1995).

[6] W. Melnitchouk, R. Ent, and C. Keppel, Phys. Rep. 406, 127 (2005).

[7] Z. E. Meziani et al., Phys. Lett. B 613, 148 (2005); K. Slifer et al., Phys. Rev. Lett. 101, 022303 (2008).

[8] X.-D. Ji and W. Melnitchouk, Phys. Rev. D 56, R1 (1997).

[9] S. Simula, M. Osipenko, G. Ricco, and M. Taiuti, Phys. Rev. D 65, 034017 (2002); M. Osipenko et al., ibid. 71, 054007 (2005).

[10] Y. Prok (CLAS), Phys. Lett. B 672, 12 (2009).

[11] P. Solvignon et al., Phys. Rev. Lett. 101, 182502 (2008).

[12] Y. B. Dong, Phys. Rev. C 77, 015201 (2008).

[13] O. Nachtmann, Nucl. Phys. B 63, 237 (1973).

[14] S. Wandzura, Nucl. Phys. B 122, 412 (1977).

[15] H. Georgi and H. D. Politzer, Phys. Rev. D 14, 1829 (1976).

[16] S. Matsuda and T. Uematsu, Nucl. Phys. B 168, 181 (1980).

[17] A. V. Sidorov and D. B. Stamenov, Mod. Phys. Lett. A 21, 1991 (2006)

[18] A. Accardi and W. Melnitchouk, Phys. Lett. B 670, 114 (2008).

[19] A. Piccione and G. Ridolfi, Nucl. Phys. B 513, 301 (1998).

[20] S. Wandzura and F. Wilczek, Phys. Lett. B 72, 195 (1977).

[21] F. R. Wesselmann (RSS), Phys. Rev. Lett. 98, 132003 (2007).

[22] M. K. Jones (RSS), Phys. Rev. C 74, 035201 (2006).

[23] P.E. Bosted and M.E. Christy, Phys. Rev. C 77, 065206 (2008); M.E. Christy and P.E. Bosted, Phys. Rev. C 81, 055213 (2010).

[24] R. Bradford, A. Bodek, H. S. Budd, and J. Arrington, Nucl. Phys. B, Proc. Suppl. 159, 127 (2006); J. Arrington, W. Melnitchouk, and J. A. Tjon, Phys. Rev. C 76, 035205 (2007).

[25] C. Ciofi degli Atti, L. P. Kaptari, S. Scopetta, and A. Y. Umnikov, Phys. Lett. B 376, 309 (1996).

[26] O. A. Rondon, Phys. Rev. C 60, 035201 (1999).

[27] S. A. Kulagin and W. Melnitchouk, Phys. Rev. C 77, 015210 (2008); Y. Kahn, W. Melnitchouk, and S. A. Kulagin, ibid. 79, 035205 (2009).

[28] K. Kramer et al., Phys. Rev. Lett. 95, 142002 (2005).

[29] I. P. Ivanov, N. N. Nikolaev, W. Schafer, and A. V. Pronyaev, Phys. Rep. 320, 175 (1999).

[30] E. V. Shuryak and A. I. Vainshtein, Nucl. Phys. B 201, 141 (1982); X. Ji and C. Chou, Phys. Rev. D 42, 3637 (1990).

[31] X.-D. Ji, W. Lu, J. Osborne, and X.-T. Song, Phys. Rev. D 62, 094016 (2000); A. V. Belitsky, X.-D. Ji, W. Lu, and J. Osborne, ibid. 63, 094012 (2001).

[32] H. Burkhardt and W. N. Cottingham, Ann. Phys. (N.Y.) 56, 453 (1970).

[33] J. Kodaira, S. Matsuda, T. Uematsu, and K. Sasaki, Phys. Lett. B 345, 527 (1995).

[34] J. D. Bjorken, Phys. Rev. 148, 1467 (1966).

[35] S. Eidelman et al., Phys. Lett. B 592, 1 (2004).

[36] A. L. Kataev, Phys. Rev. D 50, R5469 (1994).

[37] S. Choi, M. Jones, Z.-E. Meziani, and O. A. Rondon, JLab Experiment E07003; A. Camsonne, J. P. Chen, D. Crabb, and K. Slifer, JLab Experiment E08027. 